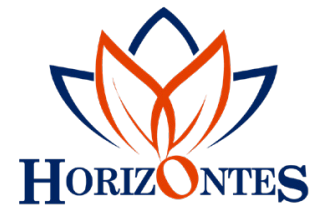

www.revistahorizontes.org
Horizontes. Revista de Investigación en Ciencias de la Educación Https://doi.org/10.33996/revistahorizontes.v5i19.245

julio-septiembre 2021

Volumen 5 / No. 19

ISSN: 2616-7964

ISSN-L: 2616-7964 pp. 892 - 900

\title{
Aplicación de las TIC en la lectura musical
}

\author{
Application of ITC in music reading
}

Aplicação das TIC na leitura de música

ARTícuLO DE INVESTIGACIÓN

\author{
Erwin Mamani Mamani \\ mamanimamanierwin@gmail.com \\ https://orcid.org/0000-0002-0035-8120
}

Universidad Púbica de El Alto, La Paz-Bolivia

\author{
Roger Quispe Chambi \\ rogercheluck@gmail.com \\ https://orcid.org/0000-0003-0640-9045 \\ Unidad Académica Lorenza Congo, \\ San Ignacio de Moxos-Bolivia
}

Recibido 4 de mayo 2021 | Arbitrado y aceptado 2 de junio 2021 | Publicado en 01 julio 2021

\section{RESUMEN}

La presente investigación aborda sobre la aplicación de herramientas y recursos tecnológicos a través del software libre Sibelius, Harmony, Finale, Lenmus y aplicaciones Android: oído perfecto, Solfa Read, para desarrollar los procesos formativos del aprendizaje de la lectura musical que se constituye en un factor determinante en la ejecución de instrumentos musicales; siendo una de las dificultades que atraviesan los estudiantes de 3er año de la especialidad de Educación Musical de la Unidad Académica de Formación de Maestros Multiétnica Lorenza Congo de la Ciudad de San Ignacio de Moxos del departamento del Beni, Bolivia. El estudio presentado tiene como objetivo determinar, porque los estudiantes presentan la dificultad de leer una partitura, siendo que se encuentran formándose para ser maestros de educación musical. Con la aplicación y uso de estos recursos y herramientas tecnológicas se ha logrado superar esas dificultades.

Palabras clave: Lectura Musical; Educación musical; Solfeo; Ejecución de instrumentos musicales; Tecnologías de información y comunicación

\section{ABSTRACT}

This research addresses the application of tools and technological resources through free software Sibelius, Harmony, Finale, Lenmus and Android applications: perfect ear, Solfa Read, to develop in the formative processes of learning to read music that constitutes a determining factor in the performance of musical instruments; being one of the difficulties experienced by 3rd year students of the specialty of Music Education of the Lorenza Congo Academic Unit for the Training of Multiethnic Teachers of the City of San Ignacio de Moxos in the department of Beni, Bolivia. The study presented below, aims to determine why students have difficulty reading a score, being that they are training to be teachers of music education. With the application and use of these resources and technological tools, these difficulties have been overcome.

Key words: Leitura musical; educação musical; solfejo; execução de instrumentos musicais; Tecnologias de informação e comunicação

\section{RESUMO}

Esta pesquisa aborda a aplicação de ferramentas e recursos tecnológicos através dos softwares livres Sibelius, Harmony, Finale, Lenmus e aplicativos Android: ouvido perfeito, Solfa Read, para desenvolver os processos formativos de aprendizagem da leitura musical que constituem um fator determinante na performance musical instrumentos; sendo uma das dificuldades enfrentadas pelos alunos do $3^{\circ}$ ano da especialidade de Educação Musical da Unidade Acadêmica de Formação de Professores Multiétnicos de Lorenza Congo da Cidade de San Ignacio de Moxos no departamento de Beni, Bolívia. O estudo apresentado a seguir, tem como objetivo verificar o motivo pelo qual os alunos têm dificuldade de leitura de uma partitura, sendo que estão em formação para serem professores de educação musical. Com a aplicação e utilização desses recursos e ferramentas tecnológicas, essas dificuldades foram superadas.

Palavras-chave: Leitura musical; educação musical; solfejo; execução de instrumentos musicais; Tecnologias de informação e comunicação 


\section{INTRODUCCIÓN}

En la actualidad, las Tecnologías de la Información y la Comunicación (de ahora en adelante TIC) están cada vez más presentes es los procesos educativos, mientras la educación sigue sin una integración de las mismas en las aulas y en el currículum, desde hace mucho tiempo al inicio del nuevo milenio (comienzos del 2000) surgieron las primeras herramientas tecnológicas en el área de educación musical como el Encore, programa que ha permitido digitalizar las partituras y generar audios de la partitura, también ha permitido que algunos maestros la incorporen en los procesos pedagógico con la finalidad de desarrollar sesiones de lectura musical, existen además, una amplia gama de recursos y herramientas tecnológicas que han permitido innovar y potenciar los aprendizajes en el área de educación musical.

Sin embargo, los procesos formativos que se desarrollan en la especialidad de Educación Musical en la Unidad Académica de Formación de Maestros Multiétnica "Lorenza Congo", los estudiantes presentan dificultades serias en el desarrollo de la lectura musical que ha generado un efecto directo a falta de conocimiento de la lectura musical, lo cual hace aún más complejo realizar la ejecución de diferentes instrumentos musicales, como trompeta, acordeón, teclado, clarinete, violín entre otros. La población estudiada son los estudiantes de tercer año de formación de educación musical de la Unidad Académica de Formación Maestros Multiétnica Lorenza Congo dependiente del Ministerio de Educación de Bolivia, realiza los procesos formativos en la Provincia Moxos, Localidad de San Ignacio de Moxos, departamento del Beni. En ese afán, se ha empezado aplicar recursos y herramientas tecnológicas que permitan ayudar a la comprensión del solfeo percusivo, hablado y entonado.

Por ello, en la educación actual son demandantes el uso nuevas metodologías educativas en las que los estudiantes sean un sujeto activo en el proceso de enseñanza y aprendizaje. Es por ello que, las TIC suponen un medio o recurso que propicia una educación activa, dinámica e interactiva donde los estudiantes puedan construir su conocimiento obteniéndolo mediante un aprendizaje significativo. En este sentido, la competencia de aprender a aprender constituye uno de los principales ejes de la educación actual, por la situación que se vive por la pandemia del COVID-19 a nivel mundial, producto de ella muchos proceso dieron pasos agigantados en el uso acelerado de las herramientas y recursos tecnológicos en la educación, dejando de lado los modelos de instrucción.

Es por ello, que el constructivismo social es un principio pedagógico (Ferdig, 2007) que juega un papel determinante en el proceso pedagógico. Este modelo usa las TIC como herramientas para el aprendizaje significativo a través de experiencias reales que provoquen la construcción del propio conocimiento. Sin embargo, cabe resaltar que las TIC deben servir como un canal de aprendizaje y no como un fin en sí mismo (Ruiz Franco, y Abella García, 2011). Por ende este estudio explica las bondades que se pueden lograr a través de software Sibelius, Finale, Lenmus, Aplicación Android como oído perfecto, SolfaRead así como indagar en el desarrollo de estrategias óptimas para el aprendizaje de la lectura musical en convivencia con la sociedad del conocimiento y la información. La 
investigación llevada a cabo es de tipo pedagógicomusical vinculando dos tipos de conocimiento: Música y TIC. Es estudio además busca responder las siguientes interrogantes: ¿Cómo trabajar la educación musical a través de las TIC? y si ¿El uso de las TIC promueve un aprendizaje significativo?

Es preciso comprender que es interpretación musical

...un intérprete debe partir con la comprensión y manejo pleno de un nuevo lenguaje escrito: el musical, que comprende una gran cantidad de parámetros (pulso, altura, duración, intensidad, color, etc.), todos ellos aplicados al uso altamente complejo de un instrumento musical. Todo este "adiestramiento" técnico va de la mano de otros aspectos que paralelamente estudian para dar forma a una lectura y a una ejecución de obras musicales, que abarca desde las más sencillas hasta las más complejas" (Orlandini Robert, 2012, p. 79).

Además algunos estudios muestran que los editores de partitura:

...tiende a ser un medio útil cuando las partituras tienen un mayor grado de dificultad y cuando los alumnos tienen menos conocimientos musicales. El hecho de que el editor muestre de una manera directa la relación entre el código escrito y su correspondencia sonora puede posibilitar que los alumnos y alumnas puedan memorizar esta relación. El que no tengan que invertir un esfuerzo extra fruto de tener que integrar mentalmente las distintas informaciones (visual y auditiva) procedentes de dos fuentes de información que no se encuentran ni física ni temporalmente integradas (partitura e instrumento), facilitaría esta memorización"(Núñez, 2016, p. 86).
Es importante destacar que para referir a las didácticas, metodologías, técnicas y herramientas para procesos educativos; líneas matriciales tecnologías de la información y la comunicación en la cualificación de procesos educativos, línea transversal a la que responde educación técnica tecnológica del área de educación musical, es necesario comprender mejor la lectura musical la cual ha sido considerada como un tipo de percepción en el que la partitura genera una información al músico que tiene la habilidad de saber leerla (Sloboda, 2005).

Existen varios fundamentos teóricos que explican la importancia de interpretar un instrumento musical; como lo es la música, ya sea está interpretada por un instrumento, cantando, bailando o escuchándola, es una herramienta fundamental en la educación de los niños debido a que su inteligencia y creatividad se desarrollan $y$ fortalecen en gran manera en edad escolar contribuyendo al desarrollo biopsicosocial.

Entonces surgen algunas preguntas, porque después de tantos años de estudio en música, 12 años en educación regular el estudiante egresado del colegio y se postula a institución superior como es el caso de ser maestro de educación musical, este no conoce la notación musical ¿fallan los métodos de enseñanza? ¿falta de esfuerzo personal de los alumnos? ¿Tan complicado es comprender la notación musical?

Esta puesta en acción del recurso, el estudio tiene como objetivo determinar porque los estudiantes presentan dificultad para leer una partitura, siendo que se encuentran formándose para ser maestros de educación musical, así como también extraer la relación entre aprendizaje significativo en la 
ejecución de instrumentos a partir de la lectura musical y TIC en la educación musical.

\section{MÉTODO}

El estudio aplicación de recursos y herramientas tecnológicas para desarrollar el aprendizaje de la lectura musical fue desarrollado en la Unidad Académica de Formación de Maestros Multiétnica Lorenza Congo en Bolivia, en el área de especialidad de Educación Musical. Fue desarrollado bajo la metodología de estudio de caso, usando como instrumento la guía de observación la cual permitió proporcionar información significativa respecto a la evolución y rendimiento de los estudiantes en el proceso de aprendizaje de la lectura musical a través de las TIC. En donde fueron considerados en las Unidades de Formación solfeo percusivo hablado y entonado; técnicas ejercicios base en el teclado y acordeón; música escolar y técnicas de teclado y acordeón. Se ha realizado la aplicación de los recursos y herramientas tecnológicas para fortalecer el aprendizaje de la lectura musical. Fueron aplicados los recursos de la aplicación de SolfaRead y oído perfecto; al mismo tiempo se ha enfatizado trabajar con editores de partitura como Sibelius, Finale y Harmony. En cada sesión de desarrollo curricular fue registrado en la guía de observación las incidencias positivas que estaban brindando los recursos y herramientas TIC.

El estudio se clasifico en cuatro fases la primera estuvo relacionada con el enfoque de la metodología indígena descolonizadora bajo la cual se desarrolló la investigación y producción de conocimientos, la segunda fue mediante la imitación el cual sirvió como el medio a través del cual se interpreta la música del contexto para recuperar y registrar los saberes y conocimientos artísticos musicales que se encuentran en comunidades de San Ignacio de Moxos en Bolivia. Todo ello, tiene un rigor investigativo en la etnomusicología, conociendo las manifestaciones culturales de la comunidad la cual se hará más accesible para aproximarse a la realidad y al objetivo propuesto; la tercera se trató de la estrategia para el diálogo con la comunidad y el recojo de la información en donde se registran las festividades y actos religiosos donde se observó las manifestaciones culturales, posteriormente se recurre a las reuniones y entrevistas con los sabios y entendidos de la música, siempre reconociéndolo materialymoralmenteparaquepuedan proporcionar información fidedigna y valiosa. Para este caso se aplicaron instrumentos y técnicas de investigación para registrar la información encontrada de la realidad de la comunidad. En la cuarta fase a través de las técnicas e instrumentos utilizados se detallan los escenarios, las características de los sujetos y la información que se espera recoger. Las fichas de registro de información del músico, celular para realizar la grabación en audio y video, fotografías, la observación fueron un recurso principal en la imitación como producto de la transmisión oral y manifestación de danza y música vinculada a la espiritualidad y la vida (Matareco, 2014). Los instrumentos de investigación que fueron usados fueron son las guías de observación, diarios de campo, registros de video y audio los cuales fueron recopilados en los diferentes escenarios vinculados al tema de música y danza momentos donde se hace visible las manifestaciones culturales de la comunidad. 


\section{RESULTADOS Y DISCUSIÒN}

En función a diagnóstico de la realidad educativa de la Unidad Académica Multiétnica Lorenza Congo, se han logrado resultados ampliamente favorables en el aprendizaje de la lectura musical, lo cual ha permitido que a los estudiantes de 3er año de formación los genere más condiciones para la ejecución de instrumentos inclusive obras más complejas. Las herramientas tecnológicas han inducido por constituirse como un recurso dinámico, lúdico porque las aplicaciones y programas de música han exigido al estudiante que deba conocer de los signo y códigos musicales que debían ser plasmados en la partitura o que debían haberse resuelto los ejercicios planteados por las aplicaciones.

Para visibilizar los resultados de manera objetiva como resultado de la aplicación de un cuestionario a los mismos estudiantes que han respondido el instrumento aplicado. Los programas editores de partitura han permitido que los estudiantes desarrollen la capacidad de introducción de partituras mediante scanner, introducción de las notas mediante micrófono, creación de partituras para ver y reproducir la obra, VST y librerias con sonidos reales, live Tempo (r) Grabación de la interpretación o ejecución en tiempo real, formatos finales del programa (PDF, WAV, TIFF, PNG, EPS, BMP, MIDI, XML).

La aplicación oído perfecto que han utilizado los estudiantes en formación es una aplicación que permite poner en práctica las habilidades auditivas relacionadas con la música. Tiene como objetivo entrenar la capacidad de reconocer intervalos, escalas y acordes. Además, incluye teoría en formato de texto con las partituras correspondientes.

El programa Sibelius y Finale utilizado por los estudiantes en formación, es un programa informático que permite escribir, ejecutar, imprimir y publicar partituras de música. Diseñado para toda clase de músicos, desde estudiantes y profesores hasta compositores profesionales, que le permite trabajar con diferentes formatos de introducción de las notas, desde grabación con dispositivos MIDI a selección con el ratón o teclado.

La aplicación que los estudiantes han utilizado como Solfa Read tiene como objetivo ayudar a mejorar su velocidad de lectura musical. Se elige una clave, un nivel e inicia el juego teniendo 2 minutos para identificar tantas notas como sea posible. Acordes y solfeo es la herramienta perfecta para tu educación musical. Este juego de música te enseña teoría musical de una forma fácil y divertida. Con esta aplicación, han comprendido los estudiantes todo lo que hay que saber sobre intervalos musicales y acordes. Si prácticas lo suficiente, podrás llegar a saber las notas contenidas en un acorde de forma instantánea.

El siguiente Gráfico 1 presenta cuán significativo fue el aporte de las TIC en el aprendizaje de la lectura musical; además, la importancia de facilitar la ejecución de los instrumentos musicales inclusive obras más trabajadas y complejas. 


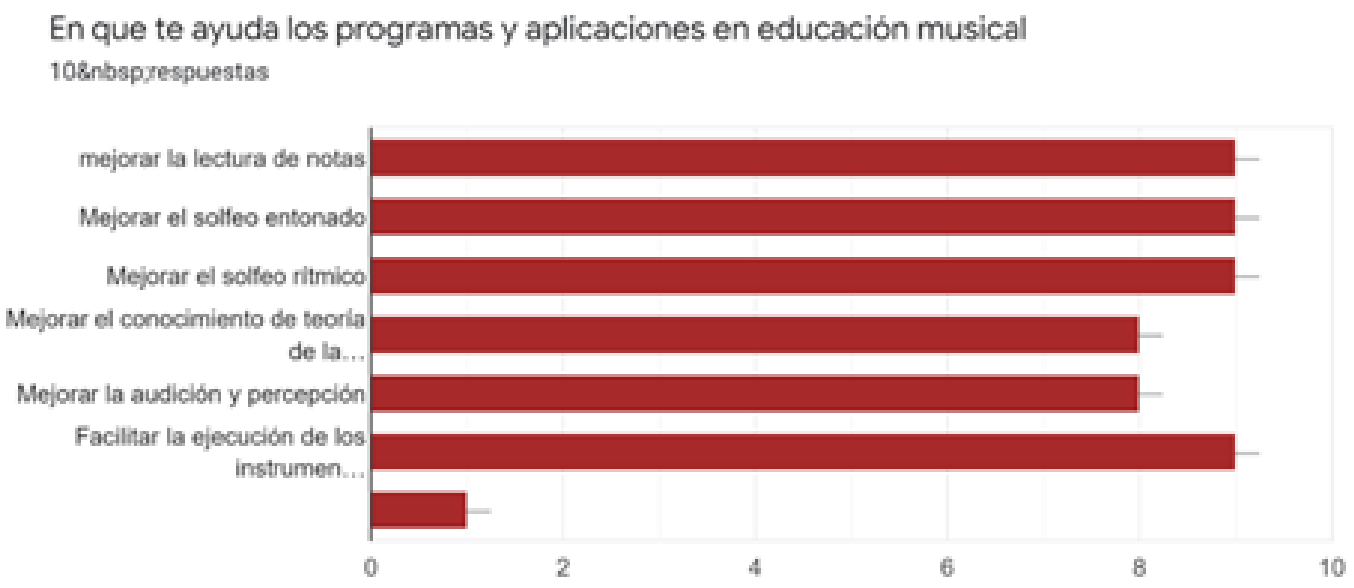

Gráfico 1. Beneficios de los programas y aplicaciones en educación musical.

Los estudiantes de la especialidad de educación musical han logrado mejorar en el solfeo percusivo, hablado y entonado, con un amplio conocimiento en la teoría de la música, mejoras en la percepción auditiva $y$ fundamentalmente con muchas condiciones para realizar la interpretación de un instrumento musical.

En el Gráfico 2 se puede observar que los estudiantes no pueden prescindir del uso de las herramientas y recursos tecnológicos en educación musical, porque su uso les trae varios beneficios intrínsecos que favorecen en el proceso formativo. En la siguiente gráfica se demarca la importancia del uso de las TIC en los procesos de aprendizaje de la lectura musical.

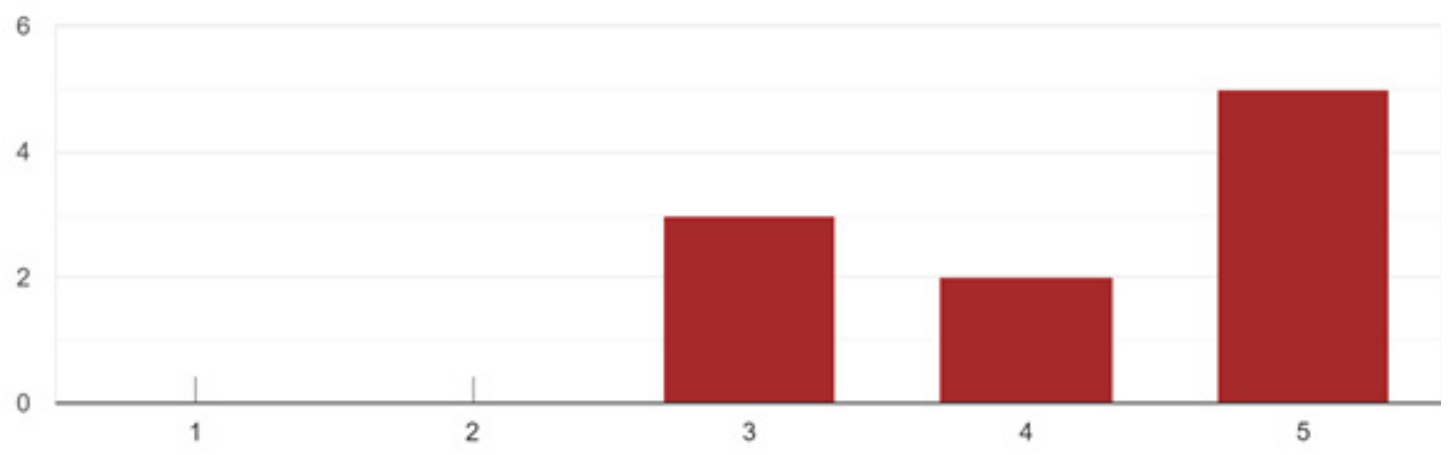

Gráfico 2. El valor y el aporte de las TIC en lectura musical. 
Es significativo el uso de las aplicaciones y programas de música, ya que ha permitido superar las falencias que tenían los estudiantes de 3er año de formación, puesto que para algunos estudiantes la unidad académica fue la primera institución donde ha empezado a conocer la notación musical y no así en la escuela o colegio donde relatan los estudiantes que solo se dedicaban a cantar y copiar himnos patrióticos. Ese vacío ha sido superado con la aplicación estratégica en las unidades de formación de especialidad que más tarde ha sido socializada con el equipo docente de la especialidad para valorar los resultados significativos que han permitido resolver el problema.

El Gráfic 3 demuestra que los estudiantes reconocen que ha incidido en gran medida los recursos y herramientas TIC que se aplicaron en las Unidades de Formación que ha facilitado la comprensión de signos y códigos musicales que les ha permitido interpretar con más accesibilidad los instrumentos musicales.

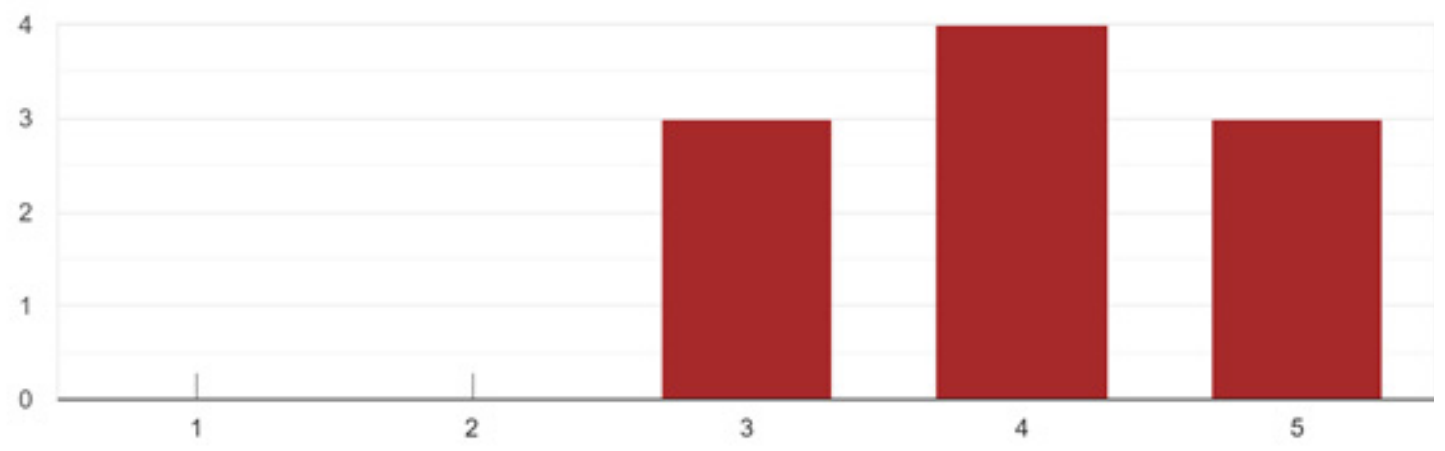

Gráfico 3. El aporte de las TIC en la Ejecución o interpretación de un instrumento musical.

Conforme a estos resultados es conveniente que se utilicen otro tipo de recursos y herramientas tecnológicas específicas para trabajar otros ámbitos que requieren de ser mejorados, como instrumentos musicales, educación auditiva, armonía, que enriquezcan aún más el bagaje musical, desarrollen ciertas habilidades, así como posibiliten un uso más flexible dentro del estudio de la lectura musical. Desde esta perspectiva se considera interesante que se destine una o dos sesiones como introducción a los editores de partituras en el contexto de la asignatura de Lectoescritura Musical, ya que el conocimiento de este tipo de programas puede hacer un mejor uso dentro de una herramienta apropiada para el estudio de la lectura musical y además, para otro tipo de actividades como los dictados musicales, la edición de partituras e incluso tareas de composición musical. La inclusión de este tipo de medios podría suponer un nuevo enfoque dentro de la asignatura en el que se pueden beneficiar los estudiantes que tienen amplios conocimientos como aquellos que tienen serias carencias. Lo expresan como un recurso imprescindible en su etapa de formación de maestros en educación musical y que también muy motivados reflejan que serán replicados en las unidades educativas donde realizan la Práctica Educativa Comunitaria y fue motivador porque de esas prácticas retornaron con motivación. 


\section{Discusión}

Una vez llevado a la práctica pedagógica la innovación con la implementación de las tecnologías de información y comunicación procederemos a analizar qué ventajas que hemos encontrado en la aplicación de las TIC en el aula de música.

Asímismo, ciertos problemas técnicos surgieron durante las sesiones que los estudiantes no pudieron solucionar algunos programas no podían abrirse porque faltaba instalar algunos componentes, cada equipo de computación tenía diferentes características lo que nos obligó a organizar por parejas en otros ordenadores, resolviendo de esa manera con la instalación de algunos drivers, códec, net frameworks entre otros.

Fue para los estudiantes más emocionante realizar transcripciones, composiciones, arreglos y registros de partitura de su preferencia, así como la música originaria que cada uno fue realizando y encontrando muchas posibilidades que les brinda estos programas como el Musescore, Finale, Sibelius, oído perfecto.

El aprendizaje de la notación musical ha sido estimulada por que indirectamente ha exigido que tengan un dominio de la teoría de la música avanzado, porque muchos estudiantes no contaban con las condiciones teóricas que deberían tener en ese nivel de estudio en educación superior, porque empezaron desde su ingreso en la UA con desconocimiento total de la teoría de la música que les ha dificultado en poder realizar estudios prácticos en la ejecución de instrumentos musicales como el acordeón, guitarra, trompeta, violín, piano, coro, orquestas entre otros.

Las herramientas y recursos tecnológicos establecen una simple explicación de cada nuevo concepto de teoría musical introducido, lo cual fue aprovechado al máximo por los estudiantes manipulando divertidos ejercicios para practicar. No importa si tu instrumento es la guitarra, el piano, el violín o cualquier otro.

La progresión gradual de la dificultad de los ejercicios y la teoría musical que presentaron los programas y aplicaciones donde las puntuaciones obtenidas en cada ejercicio se guardan para que puedas ver el estudiante su mejora, se han constituido en factores determinantes en el aprendizaje de la lectura musical.

Las capacidades y habilidades que potenciaron los estudiantes como la percepción auditiva y la incorporación de las TIC durante los procesos formativos en las Unidades de Formación de educación musical que ha favorecido el aprendizaje de la lectura musical y en consecuencia un aporte positivo contribuyendo mejores condiciones para interpretar instrumentos musicales lo cual conlleva inclusive la resolución de obras más complejas.

\section{CONCLUSIONES}

El aprendizaje a través del uso de los TIC en el proceso de la lectura musical es fundamental porque la mayoría de los estudiantes de la carrera educación musical han empezado a realizar una lectura más ligera de lo acostumbrado sin el apoyo de las TIC. Así mismo, las herramientas TIC en la especialidad de educación musical han permitido mejorar la habilidad de percepción auditiva de la altura de los sonidos, intervalos y armonía.

\section{REFERENCIAS BIBLIOGRÁFICAS}

Ferdig, R. E. (2007). Examining social software in teacher education. Journal of Technology and teacher education, 15(1), 5-10 
Matareco, E. T. (2014). El encanto de mi pueblo. Beni

Núñez, M. G. (2016). Estudio sobre la efectividad de los editores de partituras y el instrumento, en el estudio de la lectura musical cantada. REVISTA DE EDUCAÇÃO E HUMANIDADES, 79-89

Orlandini Robert, L. (2012). La interpretación musical. Revista musical chilena, 66(218), 7781.
Ruiz Franco, M., y Abella García, V. (2011). Creación de un blog educativo como herramienta TIC e instrumento TAC en el ámbito universitario.

Sloboda, J. (2005). Exploring the musical mind: Cognition, emotion, ability, function. Oxford University Press 